\title{
Prediction-based control of LTI systems with input and output time-varying delays
}

\author{
V. Léchappéa,*, E. Moulay ${ }^{\mathrm{b}}$, F. Plestan ${ }^{\mathrm{c}}$ \\ ${ }^{a}$ University of South Pacific, School of Engineering and Physics, Laucala campus, Suva, Fiji \\ ${ }^{b} X L I M$, UMR CNRS 7252, Université de Poitiers, Poitiers, France \\ ${ }^{c}$ Ecole Centrale de Nantes - LS2N, UMR CNRS 6004, Nantes, France
}

\begin{abstract}
The stability of a prediction-based controller is studied in presence of time-varying delays both in the input and in the output. Thanks to the reduction method and a Lyapunov-Krasovskii analysis, stability conditions are derived. A comparison is also made between the single input delay and single output delay cases. It is shown that this method can be applied to stabilize output delay systems without restriction on the delay rate. The results are illustrated numerically on a double integrator.
\end{abstract}

Keywords: Prediction-based control, input delay, output delay, time-varying delay, reduction method.

\section{Introduction}

Input delay and output delay systems are a subclass of time delay systems (TDS). The reader can refer to survey papers [1], [2] and books [3], [4] for a general review on TDS. Input and output delays can arise because of data acquisition or because of latencies during communications between the controller, the plant and the sensors. The latter example is particularly common for remote controlled devices such as UAVs, satellites or in Networked Control Systems (NCS). Usually input and output delays are treated similarly because they have similar effects on the system.

There exist two different approaches to control such systems: memoryless (or memory free) and memory controllers. The advantage of memory free controllers is that they do not require the computation of an integral. The reader can refer to the following articles: [5] for bounded control, [6] for adaptive control, [7] and [8] for a truncated predictor, [9] for continuous pole placement, [10] for Partial Spectrum Assignment (PSA) and [11] for sliding mode techniques. The drawback of this approach is that they usually cannot guarantee a good level of performance for unstable systems with large delays. In this case, memory controllers can be designed. For systems with a single delay (in the input or in the output), a memory controller is often a controller based on the computation of a prediction. It has been highlighted in [12] that state prediction is a fundamental concept for delay systems, much like state observation is for systems with incomplete state measurements. The most well-known method is the Smith predictor. This frequency approach was introduced by Smith

\footnotetext{
* Corresponding author

Email addresses: lechappe.vincent@gmail.com (V. Léchappé), emmanuel.moulay@univ-poitiers.fr (E. Moulay), franck.plestan@ec-nantes.fr (F. Plestan)
}

at the end of the 1950s in [13]. At the end of the 1970's and the beginning of the 1980's, the result of Smith has been extended to state-space representation and unstable systems in [14] and [15]. In [16], this approach has been extended for input, output and state delays. In [17], the standard prediction is modified to get more robustness against external disturbances. All these methods are designed for constant delays.

When the delay is time-varying, it has been shown in [18] that it is possible to perfectly compensate it but it requires the knowledge of the delay in advance. This result has been extended to nonlinear systems with both input and state delays in [19]. In practice, this is generally not possible to know the delay value in advance that is why alternative predictive techniques have been developed. In [20], the sub predictor method developed in [21] has been modified and extended to time-varying output and input delays. The advantage of this method is that it is finite dimensional. However it can not deal with arbitrarily large input or output delays. In [22], an approximate predictor (based on the constant delay predictor) is computed for time-varying delays in the input. It is shown that the stability is preserved if the delay rate is sufficiently small.

In this article, the method presented in [22] (for timevarying input delay) is extended to LTI systems with timevarying delays both in the input and the output. It is considered that the full state is known but that the measurement is delayed as well as the input. In the constant delay case, input and output delays have similar effects on the stability of the systems. As a consequence, for the time-varying delay case, it is expected that the stability conditions that hold for the input delay case will also hold for the output delay case. However, it is shown that for a time-varying output delay, no bound on the delay timederivative is required. 
The paper is organized as follows. The problem, the assumptions and the stability analysis when both input and output delays affect the system are presented in Section 2. The special cases of a single input delay and a single output delay are given in Section 3. Simulations illustrate previous theoretical results in Section 4. Finally, some perspectives are given in Section 5 .

\section{Main result}

\subsection{System presentation and assumptions}

The systems considered in this work have the following form

$$
\left\{\begin{array}{l}
\dot{x}(t)=A x(t)+B u\left(t-h_{I}(t)\right) \\
y(t)=x\left(t-h_{O}(t)\right) \\
x(\theta)=\phi_{x}(\theta) \text { for } \theta \in\left[-h_{\max }, 0\right]
\end{array}\right.
$$

where $x(t) \in \mathbb{R}^{n}, A \in \mathbb{R}^{n \times n}, B \in \mathbb{R}^{n \times p}, u(t) \in \mathbb{R}^{p}$ and $\phi_{x}$ is continuous ${ }^{1}$. The delays $h_{I}(t)$ and $h_{O}(t)$ are known, time-varying and verify the assumptions below.

Assumption 1. The delays $h_{I}(t)$ and $h_{O}(t)$ are bounded, i.e. there exist $h_{\min } \geq 0$ and $h_{\max }>0$ such that

$$
h_{\min } \leq h_{I}(t) \leq h_{\max }
$$

and

$$
h_{\min } \leq h_{O}(t) \leq h_{\max } .
$$

Assumption 2. The delays $h_{I}(t)$ and $h_{O}(t)$ are differentiable and their time derivatives are bounded, i.e. there exist $\delta_{I}>0$ and $\delta_{O}>0$ such that

$$
\left|\dot{h}_{I}(t)\right| \leq \delta_{I}
$$

and

$$
\left|\dot{h}_{O}(t)\right| \leq \delta_{O}
$$

In addition, it is assumed that the following assumption holds.

Assumption 3. The pair $(A, B)$ is stabilizable, so there exists a matrix $K$ such that $A+B K$ is Hurwitz and this ensures the existence of a symmetric positive matrix $P$, solution of the Lyapunov equation

$$
(A+B K)^{T} P+P(A+B K)=-c_{u} I_{n}
$$

with $c_{u}>0$ and $I_{n}$ the identity matrix of order $n$.

The objective is to design a prediction-based controller inspired by the constant delay case prediction and to study the influence of the time-varying delay on the closed-loop stability. In the case of a time-varying delay, it is very difficult to compute the exact prediction since it would require to know future values of the delay [18], [19]. However, this case is not very common in practice. Therefore,

\footnotetext{
${ }^{1}$ This guarantees that $\sup _{s \in\left[-h_{\max }, 0\right]}\|x(s)\|^{2}$ is well defined.
}

an approximate prediction $z(t)$ is computed thanks to the current value $h(t)=h_{I}(t)+h_{O}(t)\left(h(t) \in\left[2 h_{\min }, 2 h_{\max }\right]\right)$ as follows

$$
z(t)=e^{A h(t)} x\left(t-h_{O}(t)\right)+\int_{t-h(t)}^{t} e^{A(t-s)} B u(s) d s
$$

for all $t \geq 0$.

Remark 2.1. On top of the initial condition on $x(t)$ for $t \in\left[-h_{\max }, 0\right]$, an initial condition $u(s)=\phi_{u}(s)$ for $s \in$ $\left[-2 h_{\max }, 0\right]$ is needed to compute $z(t)$. Note that $\phi_{u}$ has to be bounded on $\left[-2 h_{\max }, 0\right]$ to ensure that $\sup _{s \in\left[-2 h_{\max }, 0\right]}\|u(s)\|^{2}$ is well defined. In addition, it is also required that $\phi_{u}$ is differentiable and that $\dot{\phi}_{u}(0)=K \dot{z}(0)$ in order to have $u$ differentiable for all $t \geq-2 h_{\max }$.

Note that $z(t)$ is an approximate prediction so the delay is not be perfectly compensated. This approximate prediction can then be used to compute the controller

$$
u(t)=K z(t)
$$

for all $t \geq 0$. The convergence analysis of the closed-loop system (1)-(8) is given in the next section.

\subsection{Convergence result}

The condition for the stability of the closed-loop system (1)-(8) is given in the following theorem.

Theorem 1. Consider system (1) which satisfies Assumptions 1, 2, 3. Suppose that system (1) is controlled by (8) with $z$ defined by (7) and define

$$
\begin{aligned}
\Upsilon_{I O}(t)= & \sup _{s \in\left[t-h_{\max }, t\right]}\|x(s)\|^{2}+\sup _{s \in\left[t-2 h_{\max }, t\right]}\|u(s)\|^{2} \\
& +\sup _{s \in\left[t-2 h_{\max }, t\right]}\|\dot{u}(s)\|^{2} .
\end{aligned}
$$

Then, there exist $\varsigma_{I O}, \varrho_{I O}, \delta_{I}^{*}>0$ such that, provided

$$
\delta_{I}<\delta_{I}^{*}
$$

one has

$$
\Upsilon_{I O}(t) \leq \varsigma_{I O} \Upsilon_{I O}(0) e^{-\varrho_{I O} t}, \quad \forall t \geq 0
$$

and therefore $\lim _{t \rightarrow+\infty}\|x(t)\|=0$.

Proof. By differentiating (7) thanks to Leibniz's rule and using (1), it can be verified that the prediction $z(t)$ is solution of the following equation

$$
\begin{aligned}
\dot{z}(t)= & A z(t)+B u(t)+\dot{h}_{I} A z(t)+\dot{h}_{I} e^{A h} B u(t-h(t)) \\
& -\dot{h}_{I} A \int_{t-h(t)}^{t} e^{A(t-s)} B u(s) d s \\
& +\left(1-\dot{h}_{O}\right) e^{A h} B \int_{t-h(t)}^{\phi(t)} \dot{u}(s) d s
\end{aligned}
$$


for all $t \geq 0$ and where $\phi(t)=t-h_{O}(t)-h_{I}\left(t-h_{O}(t)\right)$. The following Lyapunov-Krasovskii functional candidate is chosen

$$
V(t)=V_{1}(t)+V_{2}(t)+V_{3}(t)
$$

where

$$
\begin{aligned}
& V_{1}(t)=z^{T}(t) P z(t), \\
& V_{2}(t)=\int_{t-2 h_{\max }}^{t}\left(2 h_{\text {max }}+s-t\right)\|u(s)\|^{2} d s, \\
& V_{3}(t)=\int_{t-2 h_{\text {max }}}^{t}\left(2 h_{\text {max }}+s-t\right)\|\dot{u}(s)\|^{2} d s .
\end{aligned}
$$

Note that $P$ is defined in (6). The term $V_{1}$ is similar to the one used in the delay free case. The terms $V_{2}$ and $V_{3}$ are required to deal with the delayed terms in (12). In particular, $V_{3}$ is necessary to deal with the delayed term that arise because of the input/output delay combination $\left(1-\dot{h}_{0}\right) e^{A h} B \int_{t-h(t)}^{\phi(t)} \dot{u}(s) d s$. Differentiating $V_{1}(t)$ along the trajectory of (12) and using Assumptions 1, 2, 3 gives

$$
\begin{aligned}
\dot{V}_{1}(t) \leq & -c_{u}\|z(t)\|^{2}+2 \delta_{I}\|P\|\|A\|\|z(t)\|^{2} \\
& +\delta_{I} N\|z(t)\|\|u(t-h)\| \\
& +\delta_{I} M\|z(t)\| \int_{t-2 h_{\max }}^{t}\|u(s)\| d s \\
& +\left(1+\delta_{O}\right) N\|z(t)\| \int_{t-h(t)}\|\dot{u}(s)\| d s
\end{aligned}
$$

with

$$
\begin{aligned}
& N=2\|P\|\|B\| e^{2\|A\| h_{\max }} \\
& M=2\|P\|\|A\|\|B\| e^{2\|A\| h_{\max }} .
\end{aligned}
$$

Remark that the following inequality holds

$$
\|u(t-h(t))\| \leq\|K\|\|z(t)\|+q(t)
$$

where $q(t)=\int_{t-2 h_{\max }}^{t}\|\dot{u}(s)\| d s$. Thus from (18) one can show that

$$
\begin{aligned}
\dot{V}_{1}(t) \leq & -c_{u}\|z(t)\|^{2}+2 \delta_{I}\|P\|\|A\|\|z(t)\|^{2} \\
& +\delta_{I} N\|K\|\|z(t)\|^{2}+\delta_{I} N q(t)\|z(t)\| \\
& +\delta_{I} M v(t)\|z(t)\|+\left(1+\delta_{O}\right) N w(t)\|z(t)\|
\end{aligned}
$$

with $v(t)=\int_{\substack{t-2 h_{\max } \\ \max (\phi(t), t-h(t))}}^{t}\|u(s)\| d s$

and $w(t)=\int_{\min (\phi(t), t-h(t))}^{\max (\phi(t), t-h(t))}\|\dot{u}(s)\| d s$. Differentiating $V_{2}$, one gets

$$
\dot{V}_{2}(t) \leq 2 h_{\max }\|u(t)\|^{2}-\int_{t-2 h_{\max }}^{t}\|u(s)\|^{2} d s .
$$

Using Jensen's inequality, one has

$$
-\int_{t-2 h_{\max }}^{t}\|u(s)\|^{2} d s \leq-\frac{1}{2 h_{\max }} v^{2}(t)
$$

so the following inequality is verified

$$
\begin{gathered}
\dot{V}_{2}(t) \leq 2 h_{\max }\|K\|^{2}\|z(t)\|^{2}-\frac{1}{4 h_{\max }} v(t)^{2} \\
-\frac{1}{2} \int_{t-2 h_{\max }}^{t}\|u(s)\|^{2} d s .
\end{gathered}
$$

Then differentiating $V_{3}$ leads to

$$
\dot{V}_{3}(t) \leq 2 h_{\max }\|\dot{u}(t)\|^{2}-\int_{t-2 h_{\max }}^{t}\|\dot{u}(s)\|^{2} d s .
$$

Noting that

$$
-\int_{t-2 h_{\max }}^{t}\|\dot{u}(s)\|^{2} d s \leq-\int_{\min (\phi(t), t-h(t))}^{\max (\phi(t), t-h(t))}\|\dot{u}(s)\|^{2} d s
$$

and using Jensen's inequality, one obtains

$$
\begin{aligned}
-\int_{t-2 h_{\max }}^{t}\|\dot{u}(s)\|^{2} d s \leq & -\frac{1}{3 D} w(t)^{2}-\frac{1}{6 h_{\max }} q(t)^{2} \\
& -\frac{1}{3} \int_{t-2 h_{\max }}^{t}\|\dot{u}(s)\|^{2} d s
\end{aligned}
$$

with $D=\max _{t \geq 0}\left|h_{I}(t)-h_{I}\left(t-h_{O}(t)\right)\right|$. In addition, from (12), one gets the maximization

$$
\begin{aligned}
\|\dot{z}(t)\| \leq & \left(\|A+B K\|+\delta_{I}\|A\|\right. \\
& \left.+\delta_{I} e^{2\|A\| h_{\max }}\|B\|\|K\|\right)\|z(t)\| \\
& +\delta_{I} e^{2\|A\| h_{\max }}\|B\| q(t) \\
& +\delta_{I} e^{2\|A\| h_{\max }}\|A\|\|\| B \| v(t) \\
& \left(1+\delta_{O}\right) e^{2\|A\| h_{\max }}\|B\| w(t)
\end{aligned}
$$

then one can deduce that

$$
\begin{aligned}
\dot{V}_{3}(t) \leq & 2 h_{\max }\|K\|^{2}\left[c_{1}\|z(t)\|^{2}+c_{2} \delta_{I}^{2} q(t)^{2}+c_{3} \delta_{I}^{2} v(t)^{2}\right. \\
& \left.+c_{4} w(t)^{2}\right]-\frac{1}{3 D} w(t)^{2}-\frac{1}{6 h_{\max }} q(t)^{2} \\
& -\frac{1}{3} \int_{t-2 h_{\max }}^{t}\|\dot{u}(s)\|^{2} d s
\end{aligned}
$$

with

$$
\begin{aligned}
& c_{1}=4\left(\|A+B K\|+\delta_{I}\|A\|+\delta_{I} e^{2\|A\| h_{\max }}\|B\|\|K\|\right)^{2}, \\
& c_{2}=\left.4 e^{4\|A\| h_{\max }}\|B\|\right|^{2}, c_{3}=4 e^{4\|A\| h_{\max }}\|A\|^{2}\|B\|^{2}, \\
& c_{4}=4\left(1+\delta_{O}\right)^{2} e^{4\|A\||| h_{\max }}\|B\|^{2} .
\end{aligned}
$$

Thus using the completing the square method [23] and Young's inequality [24] to get rid of the crossed terms, we 
have

$$
\begin{aligned}
& \dot{V}(t)+\varrho_{I O} V(t) \leq-\left[c_{u}-2 \delta_{I}\|P\|\|A\|-2 h_{\max }\|K\|^{2}-\varrho_{I O}\|P\|\right. \\
& \left.-2 h_{\max }\|K\|^{2} c_{1}-\delta_{I} N\|K\|-\delta_{I} \frac{M+N}{2}-\frac{3\left(1+\delta_{O}\right)^{2} N^{2} D}{2 \theta}\right]\|z(t)\|^{2} \\
& -\left[\frac{1}{4 h_{\max }}-2 h_{\max }\|K\|^{2} c_{3} \delta_{I}^{2}-\delta_{I} \frac{M}{2}\right] v(t)^{2} \\
& -\left[\frac{1}{3 D}-2 h_{\max }\|K\|^{2} c_{4}\right] w(t)^{2} \\
& -\left[\frac{1}{6 h_{\max }}-\delta_{I} \frac{N}{2}-2 h_{\max }\|K\|^{2} c_{2} \delta_{I}^{2}\right] q(t)^{2} \\
& -\left[\frac{1}{2}-2 \varrho_{I O} h_{\max }\right] \int_{t-2 h_{\max }}^{t}\|u(s)\|^{2} d s \\
& -\left[\frac{1}{3}-2 \varrho_{I O} h_{\max }\right] \int_{t-2 h_{\max }}\|\dot{u}(s)\|^{2} d s
\end{aligned}
$$

with $\varrho_{I O}>0$. Then it is sufficient to have negative coefficients multiplying the quadratic terms in order to make $\dot{V}(t)+\varrho_{I O} V(t) \leq 0$. Noting that from the Taylor-Lagrange formula [25], there exists $\xi \in\left[t-h_{O}(t), t\right]$ such that

$$
h_{I}\left(t-h_{O}(t)\right)=h_{I}(t)-h_{O}(t) \dot{h}_{I}(\xi)
$$

one deduces that $D \leq h_{\max } \delta_{I}$. By studying the behaviour of the coefficients multiplying the quadratic terms, it follows that it is always possible to chose $c_{u}$ sufficiently large and $\delta_{I}$ sufficiently small to get

$$
\dot{V}(t) \leq-\varrho_{I O} V(t)
$$

Computation details to obtain inequality (11) are provided in Appendix A.

From (11), it can be said that the closed-loop system (1)-(8) is exponentially stable in terms of the norm $\Upsilon_{I O}$ and that the convergence of $\|x\|$ to zero is exponential.

Remark 2.2. A bound $\delta_{I}^{*}$ could be explicitly computed from (28). However, its expression is intricate and the value will be very conservative because of the basic choice of the Lyapunov-Krasovskii functional. Some more advanced choice of Lyapunov-Krasovskii functionals as the one proposed in [26] could be used to reduce conservatism.

Remark 2.3. Since equation (12) involves the integral of $\dot{u}$, it is not possible to use a Lyapunov-Razumikhin function of the form $z^{T}(t) P z(t)$. Indeed, in order to bound

$\int_{-h(t)}^{\phi(t)} \dot{u}(s) d s$, one needs to maximize $\|\dot{z}\|$ which itself depends on $\dot{u}$ so this would lead to a recursive problem.

When time-varying input and output delays appear simultaneously in the control loop, the stability condition depends only on the input delay not on the output delay. It means that the delay $h_{I}$ has to be sufficiently slow-varying in order to preserve the closed-loop stability. Thus, it is interesting to note that as soon as the input delay is slow enough the output delay can be arbitrarily fast.

Note that it could have been possible to use $t-\phi(t)$ instead of $h(t)$ in the definition of (7) in order to cancel the term $\int_{t-h(t)}^{\phi(t)} \dot{u}(s) d s$ in the reduced system (12). Indeed, defining

$$
\bar{z}(t)=e^{A(t-\phi(t))} x\left(t-h_{O}(t)\right)+\int_{\phi(t)}^{t} e^{A(t-s)} B u(s) d s
$$

the reduced system becomes

$$
\begin{aligned}
\dot{\bar{z}}(t)= & A \bar{z}(t)+B u(t)+(1-\dot{\phi}) A \bar{z}(t) \\
& -(1-\dot{\phi}) A \int_{\phi(t)}^{t} e^{A(t-s)} B u(s) d s \\
& +(1-\dot{\phi}) e^{A(t-\phi(t))} B u(\phi(t)) .
\end{aligned}
$$

However, in this case, the terms that depend on $\dot{h}_{O}$ (that appears through $\dot{\phi}$ ) cannot be maximized without adding the extra condition that $\left|\dot{h}_{O}\right|$ is sufficiently small. That is why the prediction with $h(t)=h_{I}(t)+h_{O}(t)$ has been preferred since it only requires a condition on the input delay rate and not on the output delay rate. In addition, the advantage of the definition of $z(t)$ in $(7)$ over $\bar{z}(t)$ is that only the knowledge of the lumped delay $h(t)$ is required; the individual delays $h_{I}$ and $h_{O}$ are not required to be known.

\section{Two special cases}

In this section, two special cases of the previous results are studied. The input delay delay case is presented in Subsection 3.1 and the output delay is analyzed in Subsection 3.2. Note that a similar result has been presented in [22] for the single input case. In the subsections below, systems and delays verify Assumptions 1, 2 and 3.

\subsection{Input delay case}

When only an input delay is present, system (1) becomes

$$
\left\{\begin{array}{l}
\dot{x}(t)=A x(t)+B u\left(t-h_{I}(t)\right) \\
y(t)=x(t) \\
x(0)=x_{0}
\end{array}\right.
$$

In this case, prediction (7) is rewritten as follows

$$
z(t)=e^{A h_{I}(t)} x(t)+\int_{t-h_{I}(t)}^{t} e^{A(t-s)} B u(s) d s
$$

for all $t \geq 0$. Note that as in Section 2, $z(t)$ is an approximate prediction so the delay is not be perfectly compensated. This approximate prediction can then be used to compute the controller

$$
u(t)=K z(t)
$$

for all $t \geq 0$. The condition for the stability of the closedloop system (32)-(34) is given in the following corollary. 
Corollary 1. Consider system (32) which satisfies Assumption 3 and $h_{I}(t)$ that verifies inequalities (2) and (4). Suppose that system (32) is controlled by (34) with $z$ defined by (33) and define

$$
\Upsilon_{I}(t)=\|x(t)\|^{2}+\sup _{s \in\left[t-h_{\max }, t\right]}\|u(s)\|^{2} .
$$

Then, there exist $\varsigma_{I}, \varrho_{I}, \delta_{I}^{*}>0$ such that, provided

$$
\delta_{I}<\delta_{I}^{*}
$$

one has

$$
\Upsilon_{I}(t) \leq \varsigma_{I} \Upsilon_{I}(0) e^{-\varrho_{I} t}, \quad \forall t \geq 0
$$

and therefore $\lim _{t \rightarrow+\infty}\|x(t)\|=0$.

The proof of Corollary 1 follows the same steps as the proof of Theorem 1 and thus is omitted. Note that for convenience the same notation $\delta_{I}^{*}$ is used for the bound of the time-derivative of the input delay in Theorem 1 and Corollary 1 but their values can be different.

Remark 3.1. In [22], the delayed input is represented by a partial differential equation (PDE) driven by the input at its boundary. As a consequence, system (32) is modeled as an ordinary differential equation (ODE) cascaded with a PDE. Then a so-called "backstepping transformation" is used to simplify the boundary condition.

\subsection{Output delay case}

When only an output delay is present, system (1) becomes

$$
\left\{\begin{array}{l}
\dot{x}(t)=A x(t)+B u(t) \\
y(t)=x\left(t-h_{O}(t)\right) \\
x(\theta)=\phi_{x}(\theta) \text { for } \theta \in\left[-h_{\max }, 0\right]
\end{array}\right.
$$

In this case, prediction (7) is rewritten as follows

$$
z(t)=e^{A h_{O}(t)} x\left(t-h_{O}(t)\right)+\int_{t-h_{O}(t)}^{t} e^{A(t-s)} B u(s) d s
$$

for all $t \geq 0$. Note that in this case, $z(t)$ is the exact prediction. This prediction can then be used to compute the controller

$$
u(t)=K z(t)
$$

for all $t \geq 0$. The convergence analysis of the closed-loop system (38)-(40) is given below.

Corollary 2. Consider system (38) which satisfies Assumption 3 and $h_{O}(t)$ that verifies inequality (3). Suppose that system (38) is controlled by (40) with $z$ defined by (39) and define

$$
\Upsilon_{O}(t)=\sup _{s \in\left[t-h_{\max }, t\right]}\|x(s)\|^{2}+\sup _{s \in\left[t-h_{\max }, t\right]}\|u(s)\|^{2} .
$$

Then, there exist $\varsigma_{O}$, @o such that one has

$$
\Upsilon_{O}(t) \leq \varsigma_{O} \Upsilon_{O}(0) e^{-\varrho O t}, \quad \forall t \geq 0
$$

and therefore $\lim _{t \rightarrow+\infty}\|x(t)\|=0$.
The proof of Corollary 2 is straightforward noting that $z(t)=x(t)$ and is omitted.

Note that in the output delay case the delay $h_{O}$ does not need to be differentiable and no restriction on the delay rate is required. It means that the predictive method can deal with fast delays even disordered information. This is a direct consequence that the prediction (39) is not an approximation, as in the input delay case, but is the exact prediction. That is why it is possible to compensate perfectly for the time-varying delay. In the input delay case, a condition on the delay rate is necessary to preserve the stability as explained in Corollary 1. In Corollary 2 it is shown that no more condition on the delay rate is required. This is contrary to the intuition that input delay and output delay have the same effect on the system ${ }^{2}$.

A simulation comparison between the three cases of Sections 2 and Subsections 3.1 and 3.2 is given in the next section.

\section{Simulations}

Consider the double integrator with both input and output delays

$$
\left\{\begin{array}{l}
\dot{x}(t)=A x(t)+B u\left(t-h_{I}(t)\right) \\
y(t)=x\left(t-h_{O}(t)\right)
\end{array}\right.
$$

with input delay only

$$
\left\{\begin{array}{l}
\dot{x}(t)=A x(t)+B u\left(t-h_{I}(t)\right) \\
y(t)=x(t)
\end{array}\right.
$$

with output delay only

$$
\left\{\begin{array}{l}
\dot{x}(t)=A x(t)+B u(t) \\
y(t)=x\left(t-h_{O}(t)\right)
\end{array}\right.
$$

where $A=\left[\begin{array}{ll}0 & 1 \\ 0 & 0\end{array}\right]$ and $B=\left[\begin{array}{c}0 \\ 0.7\end{array}\right]$. Remark that Assumption 3 is verified. The state feedback

$$
u(t)=K z(t)
$$

is used to control previous systems. Controller (46) with $z$ defined by (7), (respectively (33) and (39)) is used to control (43) (respectively (44) and (45)). The same arbitrary value $K=[-6.1,-5.7]$ is chosen for the three cases in order to perform a fair comparison. Different values of the delays are used in order to illustrate Theorem 1 and Corollaries 1 and 2. These values are given in Table 1 . In each case, Assumptions 1, 2 and 3 are satisfied with $h_{\min }=0.2$ and $h_{\max }=2$. For simplicity, the initial conditions for the state and the input are taken equal to zero.

Remark 4.1. The computation of $z$ requires an integration. For open-loop stable systems, the integral term can

\footnotetext{
${ }^{2}$ This is only true when the input and output delays are constant.
} 


\begin{tabular}{c|c}
\hline Figure & Delay (sec) \\
\hline Fig. 1 & $h_{I}(t)=h_{O}(t)=1+0.5 \sin (0.5 t)$ \\
\hline Fig. 2 & $h_{I}(t)=h_{O}(t)=1+0.5 \sin (2.4 t)$ \\
\hline Fig. 3 & $\begin{array}{c}h_{I}(t)=0.5+0.4 \sin (t) \\
h_{O}(t)=0.5+0.45 \sin (25 t)\end{array}$ \\
\hline
\end{tabular}

Table 1: Different delay values tested in simulation from constant to fast varying delays

be computed without discretizing the integral [27]. However, for open-loop unstable systems, the integral has to be discretized in a finite number of points. This step has to be done very carefully since it can destabilize the system as pointed out in [28]. Safe implementations of the prediction are given in [29] and [4]. In this article, we have used a time-domain approximation with sample-and-hold that guarantee the accuracy of the prediction if the sample time is sufficiently small [30].

First, a slow-varying delay is applied to systems (44) and (45). We can see on Figure 1 that both systems are stable in spite of the time-varying delay. One can observe a transient phase where both curves are mixed up because the input is equal to zero. Then, the trajectories are different because the control inputs applied at instant $t$ are not equal anymore.
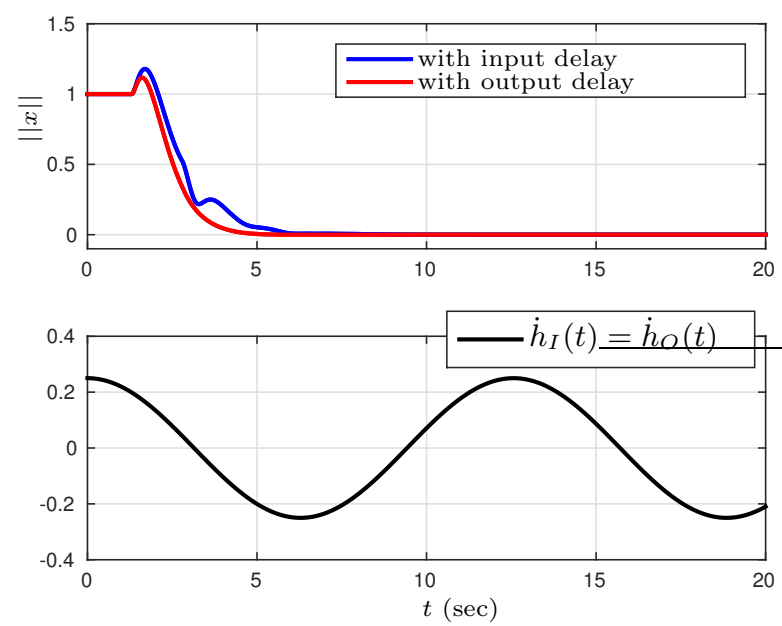

Figure 1: Comparison input and output delays prediction-based control for slow-varying delays $\left(\delta_{I}=0.25\right)$.

On Figure 2, the delay rate has been increased. In this case, it is clear that the input delay system is unstable. However, the output delay system is still stable. In addition, the state trajectory of system (45) is very similar whatever the delay value (Figures 1,2). This is in accordance with theoretical results of the previous sections. Indeed, it is stated in Corollary 1 that an input delay system with a prediction-based controller (33)-(34) becomes unstable if the delay is too fast-varying. On the contrary, Corollary 2 guarantees that an output delay system with a prediction-based controller (39)-(40) is stable whatever the delay rate.
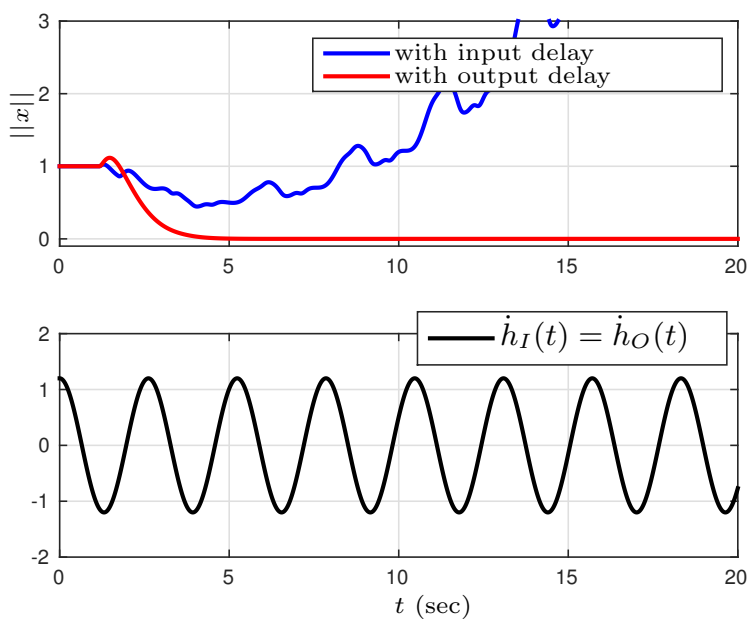

Figure 2: Comparison input and output delays prediction-based control for fast-varying delays $\left(\delta_{I}=1.2\right)$.

On Figure 3, both input and output time-varying delays are present in the loop. It can be observed that system (43) is stable in presence of a slow-varying input delay and a very fast-varying output delay. This is coherent with Theorem 1 .

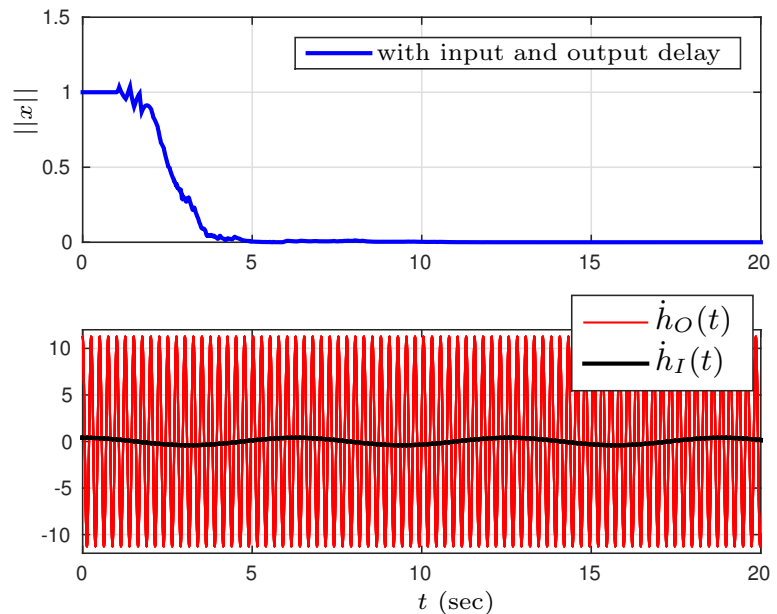

Figure 3: Input and output delays prediction-based control $\left(\delta_{I}=0.4\right.$, $\left.\delta_{O}=11.25\right)$.

\section{Conclusion}

When both input and output delays are present in the loop, the stability is preserved for arbitrarily large delays if the input delay is sufficiently slow and if the dynamics of the output delay is bounded (but there is no restriction on the size of the bound). Therefore, this method is able to preserve the stability of output delay systems for fastvarying delays. In the single input delay case, it is shown 
that the closed-loop stability is ensured provided that the delay is sufficiently slow-varying. On the contrary, no restriction on delay rate is required for closed-loop stability in presence of a single output delay. The reduction method and a Lyapunov-Krasovskii analysis are used to study the closed-loop stability. Theoretical results are illustrated by simulation on a delayed double integrator. The extension to perturbed systems using the result of [17] is considered for future works as well as a stability analysis which does not rely on reduced systems in order to get rid of the differentiability assumption of the delays.

\section{References}

[1] J.-P. Richard, Time-delay systems: an overview of some recent advances and open problems, Automatica 39 (10) (2003) 16671694.

[2] K. Gu, S.-I. Niculescu, Survey on recent results in the stability and control of time-delay systems, Journal of dynamic systems, measurement, and control 125 (2) (2003) 158-165.

[3] W. Michiels, S.-I. Niculescu, Stability and stabilization of timedelay systems: an eigenvalue-based approach, Vol. 12 of Advances in Design and Control, SIAM, 2007.

[4] Q.-C. Zhong, Robust control of time-delay systems, 1st Edition, Springer-Verlag London, 2006.

[5] F. Mazenc, S. Mondié, S.-I. Niculescu, Global asymptotic stabilization for chains of integrators with a delay in the input, IEEE Trans. Autom. Control 48 (1) (2003) 57-63.

[6] H.-L. Choi, J.-T. Lim, Stabilization of a chain of integrators with an unknown delay in the input by adaptive output feedback, IEEE Trans. Autom. Control 51 (8) (2006) 1359-1363.

[7] B. Zhou, Z. Lin, G.-R. Duan, Truncated predictor feedback for linear systems with long time-varying input delays, Automatica 48 (10) (2012) 2387-2399.

[8] S. Y. Yoon, Z. Lin, Truncated predictor feedback control for exponentially unstable linear systems with time-varying input delay, Systems and Control Letters 62 (10) (2013) 837-844.

[9] W. Michiels, K. Engelborghs, P. Vansevenant, D. Roose, Continuous pole placement for delay equations, Automatica 38 (5) (2002) 747-761.

[10] F. Cacace, A. Germani, C. Manes, Exponential stabilization of linear systems with time-varying delayed state feedback via partial spectrum assignment, Systems \& Control Letters 69 (2014) 47-52.

[11] J.-P. Richard, F. Gouaisbaut, W. Perruquetti, Sliding mode control in the presence of delay, Kybernetica 37 (3) (2001) 277294.

[12] L. Mirkin, N. Raskin, Every stabilizing dead-time controller has an observer-predictor-based structure, Automatica 39 (10) (2003) 1747-1754.

[13] O. J. M. Smith, Closer control of loops with dead time, Chemical Engineering Progress 53 (5) (1957) 217-219.

[14] A. W. Olbrot, Stabilizability, detectability, and spectrum assignment for linear autonomous systems with general time delays, IEEE Trans. Autom. Control 23 (5) (1978) 887-890.

[15] Z. Artstein, Linear systems with delayed controls: A reduction, IEEE Trans. Autom. Control 27 (4) (1982) 869-879.

[16] S. Y. Yoon, Z. Lin, Predictor based control of linear systems with state, input and output delays, Automatica 53 (2015) 385391.

[17] V. Léchappé, E. Moulay, F. Plestan, A. Glumineau, A. Chriette, New predictive scheme for the control of LTI systems with input delay and unknown disturbances, Automatica 52 (2) (2015) 179-184.

[18] M. T. Nihtila, Adaptive control of a continuous-time system with time-varying input delay, Systems \& Control Letters 12 (4) (1989) 357-364.
[19] M. Krstic, Delay compensation for nonlinear, adaptive, and PDE systems, Systems and Control: Fundations and Applications, Birkhäuser Boston, 2009.

[20] F. Cacace, A. Germani, C. Manes, Predictor-based control of linear systems with large and variable measurement delays, International Journal of Control 87 (4) (2014) 704-714.

[21] M. Najafi, S. Hosseinnia, F. Sheikholeslam, M. Karimadini, Closed-loop control of dead time systems via sequential subpredictors, International Journal of Control 86 (4) (2013) 599609.

[22] D. Bresch-Pietri, Robust control of variable time-delay systems. theoretical contributions and application to engine control, Ph.D. thesis, MINES ParisTech (2012).

[23] R. Narasimhan, Precalculus: Building concepts and connections, Houghton Mifflin, 2009.

[24] D. S. Mitrinović, J. Pečarić, A. M. Fink, Classical and new inequalities in analysis, Vol. 61, Springer Science \& Business Media Dordrecht, 2013.

[25] M. Kline, Calculus: an intuitive and physical approach, Courier Corporation New York, 1998.

[26] A. Seuret, F. Gouaisbaut, Wirtinger-based integral inequality: Application to time-delay systems, Automatica 49 (9) (2013) 2860-2866.

[27] K. Watanabe, M. Ito, A process-model control for linear systems with delay, IEEE Trans. Autom. Control 26 (6) (1981) 12611269.

[28] V. Van Assche, M. Dambrine, J.-F. Lafay, J.-P. Richard, Some problems arising in the implementation of distributed-delay control laws, in: Conference on Decision and Control, Phoenix, USA, 1999.

[29] S. Mondié, W. Michiels, Finite spectrum assignment of unstable time-delay systems with a safe implementation, IEEE Trans. Autom. Control 48 (2003) 2207-2212.

[30] B. Castillo-Toledo, S. D. Gennaro, G. S. Castro, On the problem of stabilization for linear systems with time-delays, in: American Control Conference, Montreal, Canada, 2012, pp. 46914696 .

\section{Appendix A. Obtaining inequality (11)}

From (29), one deduce that $V(t) \leq V(0) e^{-\varrho_{I O} t}$ and since $\|z(t)\|^{2} \leq \frac{1}{\lambda_{\min }(P)} V(t)$, one obtains

$$
\|z(t)\|^{2} \leq \frac{1}{\lambda_{\min }(P)} V(0) e^{-\varrho_{I} t}
$$

where $\lambda_{\min }(P)$ denotes the smallest eigenvalue of $P$. From (7), the following maximization can be deduced

$$
\|z(t)\| \leq c_{5}\left\|x\left(t-h_{O}(t)\right)\right\|+c_{6} \sup _{s \in\left[t-2 h_{\max }, t\right]}\|u(s)\| \text { (A.2) }
$$

with $c_{5}=e^{2\|A\| h_{\max }}$ and $c_{6}=2 h_{\max } e^{2\|A\| h_{\max }}\|B\|$. As a result one gets

$$
\|z(t)\| \leq c_{5} \sup _{s \in\left[t-h_{\max }, t\right]}\|x(s)\|+c_{6} \sup _{s \in\left[t-2 h_{\max }, t\right]}\|u(s)\|
$$

SO

$$
\|z(t)\|^{2} \leq 2 c_{5}^{2} \sup _{s \in\left[t-h_{\max }, t\right]}\|x(s)\|^{2}+2 c_{6}^{2} \sup _{s \in\left[t-2 h_{\max }, t\right]}\|u(s)\|^{2}
$$

and finally

$$
\|z(t)\|^{2} \leq c_{7} \Upsilon_{I O}(t)
$$


with $c_{7}=\max \left(2 c_{5}^{2}, 2 c_{6}^{2}\right)$. From (13), the maximization

$$
\begin{aligned}
V(t) \leq & \lambda_{\max }(P)\|z(t)\|^{2}+4 h_{\max }^{2} \sup _{s \in\left[t-2 h_{\max }, t\right]}\|u(s)\|^{2} \\
& +4 h_{\max }^{2} \sup _{s \in\left[t-2 h_{\max }, t\right]}\|\dot{u}(s)\|^{2}
\end{aligned}
$$

holds so

$$
V(t) \leq c_{8} \Upsilon_{I O}(t)
$$

where $c_{8}=\max \left(\lambda_{\max }(P) c_{7}, 4 h_{\max }^{2}\right)$ and $\lambda_{\max }(P)$ denotes the largest eigenvalue of $P$. Combining (A.1) with (A.7) leads to

$$
\|z(t)\|^{2} \leq c_{9} \Upsilon_{I O}(0) e^{-\varrho_{I O} t}
$$

for all $t \geq 0$ and with $c_{9}=\frac{c_{8}}{\lambda_{\min }(P)}$. Furthermore, one has

$$
\begin{aligned}
& \sup _{s \in\left[t-2 h_{\max }, t\right]}\|u(s)\|^{2} \leq \\
& \begin{cases}\sup _{s \in\left[-2 h_{\max }, 0\right]}\left\|\phi_{u}(s)\right\|^{2}+\|K\|^{2} \sup _{s \in[0, t]}\|z(s)\|^{2} & t<2 h_{\max } \\
\|K\|^{2} \sup _{s \in\left[t-2 h_{\max }, t\right]}\|z(s)\|^{2} & t \geq 2 h_{\max }\end{cases}
\end{aligned}
$$

where $\phi_{u}$ is the initial condition on $u$ defined in Remark 2.1. Note that $\phi_{u}$ is bounded so $\sup _{s \in\left[-2 h_{\max }, 0\right]}\left\|\phi_{u}(s)\right\|^{2}$ is well defined and $\sup _{s \in\left[-2 h_{\max }, 0\right]}\left\|\phi_{u}(s)\right\|^{2} \leq \Upsilon_{I}(0)$. Thus from (A.8), one has

$$
\begin{aligned}
& \sup _{s \in\left[t-2 h_{\max }, t\right]}\|u(s)\|^{2} \leq \\
& \begin{cases}\left(1+\|K\|^{2} c_{9}\right) \Upsilon_{I O}(0) & \text { if } t<h_{\max } \\
\|K\|^{2} c_{9} \Upsilon_{I O}(0) e^{2 \varrho_{I O} h_{\max }} e^{-\varrho_{I O} t} & \text { if } t \geq 2 h_{\max }\end{cases}
\end{aligned}
$$

As a consequence, choosing $c_{10}=\left(1+\|K\|^{2} c_{9}\right) e^{2 \varrho_{I O} h_{\max }}$ guarantees that

$$
\sup _{s \in\left[t-2 h_{\max }, t\right]}\|u(s)\|^{2} \leq c_{10} \Upsilon_{I O}(0) e^{-\varrho_{I O} t}
$$

for all $t \geq 0$ since $\left(1+\|K\|^{2} c_{9}\right) \Upsilon_{I O}(0) \leq c_{10} \Upsilon_{I O}(0) e^{-\varrho_{I O} t}$ for all $0 \leq t<2 h_{\max }$ and that

$\|K\|^{2} c_{9} \Upsilon_{I O}(0) e^{2 \varrho_{I O} h_{\max }} e^{-\varrho_{I O} t} \leq c_{10} \Upsilon_{I O}(0) e^{-\varrho_{I O} t}$ for all $t \geq 2 h_{\max }$. Rearranging $(7)$ gives

$$
x\left(t-h_{O}(t)\right)=e^{-A h(t)} z(t)-\int_{t-h(t)}^{t} e^{A(t-h(t)-s)} B u(s) d s .
$$

By the same steps as in (A.4), one gets

$$
\left\|x\left(t-h_{O}\right)\right\|^{2} \leq 2 c_{5}^{2}\|z(t)\|^{2}+2 c_{6}^{2} \sup _{s \in\left[t-2 h_{\max }, t\right]}\|u(s)\|^{2}
$$

for all $t \geq h_{\max }$. Inequalities (A.8) and (A.11) ensure that

$$
\left\|x\left(t-h_{O}(t)\right)\right\|^{2} \leq c_{11} \Upsilon_{I O}(0) e^{-\varrho_{I O} t}
$$

for all $t \geq h_{\max }$ with $c_{11}=\max \left(2 c_{5}^{2} c_{9}, 2 c_{6}^{2} c_{10}\right)$. A similar argument as in (A.9)-(A.10) guarantees the existence of $c_{12}>0$ such that

$$
\left\|x\left(t-h_{O}(t)\right)\right\|^{2} \leq c_{12} \Upsilon_{I O}(0) e^{-\varrho_{I O} t}
$$

for all $t \geq 0$. Then using the expression of $x(t)$ as a function of $x\left(t-h_{O}(t)\right)$ one can conclude that there exists $c_{13}>0$ such that

$$
\sup _{s \in\left[t-h_{\max }, t\right]}\|x(s)\|^{2} \leq c_{13} \Upsilon_{I O}(0) e^{-\varrho_{I O} t}
$$

for all $t \geq 0$. Since $\dot{u}(t)=K \dot{z}(t)$, one can show from (12) and using (A.8), (A.11) that there exists $c_{14}>0$ such that

$$
\sup _{s \in\left[t-2 h_{\max }, t\right]}\|\dot{u}(s)\|^{2} \leq c_{14} \Upsilon_{I O}(0) e^{-\varrho_{I O} t}
$$

for all $t \geq 0$. Finally, from (A.11), (A.16) and (A.17), one obtains

$$
\Upsilon_{I O}(t) \leq \varsigma_{I O} \Upsilon_{I O}(0) e^{-\varrho_{I O} t}
$$

for all $t \geq 0$ with $\varsigma_{I O}=\max \left(c_{10}, c_{13}, c_{14}\right)$. 